\title{
LIVER
}

\section{New biomarker identified for nonalcoholic steatohepatitis}

For patients with nonalcoholic fatty liver disease, it is important to differentiate individuals with steatosis from those with steatohepatitis as the latter are at risk of cirrhosis and hepatocelluar carcinoma and might need further treatment. However, currently, nonalcoholic steatohepatitis (NASH) can only be diagnosed with a liver biopsy, which is a high-risk procedure. A group from the USA has recently found that non-HDL cholesterol (non-HDL-C) could be used as a noninvasive biomarker for NASH, as it is easily calculated from standard lipid panels.

"Recent data have indicated that important differences in lipid metabolism may exist between patients with steatosis and NASH," explains lead author Kathleen E. Corey. Levels of non-HDL-C are simple to calculate and could be used as an indication of these differences. "Therefore, we sought to evaluate the value of nonHDL-C as a biomarker for NASH," says Corey.

The researchers enrolled 218 patients who were either undergoing liver biopsy for suspected fatty liver disease or were undergoing weight-loss surgery that included a liver biopsy. Of these patients, 100 had steatosis and 118 had NASH. Corey and colleagues then assessed the differences in serum levels of non-HDL-C between the patients. The findings were validated in a separate cohort of patients with fatty liver disease.

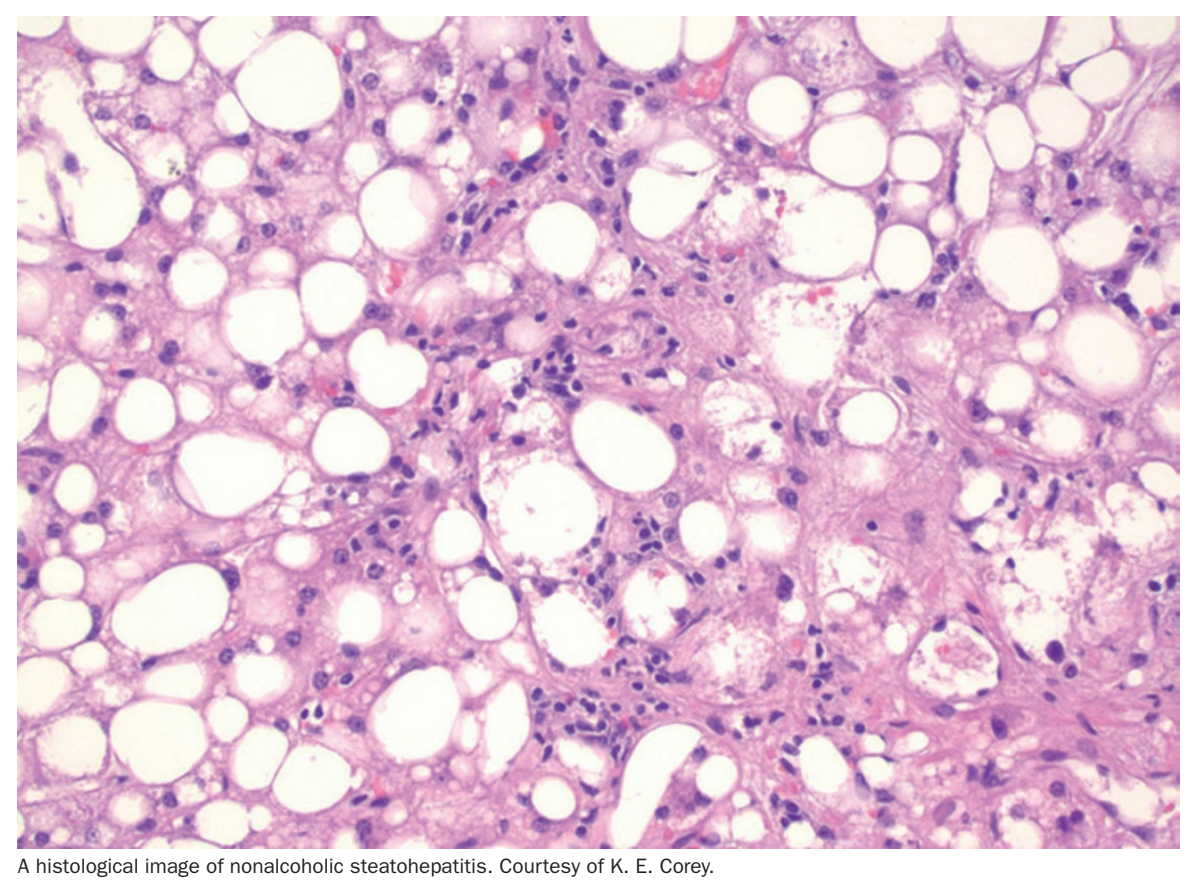

Patients with NASH showed a trend towards higher levels of non-HDL-C than those with steatosis. This difference was statistically significant in a subgroup of patients who were not taking lipidlowering medication. Thus, nonHDL-C seems to be a novel, simple and inexpensive biomarker that can be used to noninvasively diagnose NASH.

The researchers suggest that the next step will be to combine non-HDL-C with other biomarkers to improve its accuracy. "In addition, it will be important to evaluate the individual components of non-HDL-C to determine which vary between steatosis and NASH and, by extension, which may be targeted for NASH therapy," concludes Corey.

\section{Claire Greenhill}

Original article Corey, K. E. et al. Non-high density lipoprotein cholesterol as a biomarker for nonalcoholic steatohepatitis. Clin. Gastroenterol. Hepatol. doi:10.1016/j.cgh.2012.01.017 